\title{
VALIDAÇÃO DE UM INSTRUMENTO QUE CLASSIFICA OS PACIENTES EM QUATRO GRAUS DE DEPENDÊNCIA DO CUIDADO DE ENFERMAGEM
}

\author{
Eleine Aparecida Penha Martins*
}

Maria do Carmo Lourenço Haddad**

MARTINS, E.A.P.; HADDAD, M.do C.L. Validação de um instrumento que classifica os pacientes em quatro graus de dependência do cuidado de enfermagem. Rev.latino-am.enfermagem, Ribeirão Preto, v. 8, n. 2, p. 74-82, abril 2000 .

Esta pesquisa apresenta o processo de validação de um instrumento que classifica os pacientes em quatro graus de dependência do cuidado de enfermagem realizado em um hospital universitário público. O estudo proporcionou aos enfermeiros participantes a oportunidade para analisar as necessidades dos pacientes, tornando-se o instrumento uma ferramenta de trabalho eficiente na prática de enfermagem.

UNITERMOS: cuidados de enfermagem, pacientes, questionários

\section{INTRODUÇÃO}

O serviço de enfermagem, no seu dia-a-dia, encontra uma série de dificuldades relacionadas a aspectos quantitativos e qualitativos na administração de recursos, sejam eles, humanos, materiais ou físicos.

Segundo ROMERO et al. (1994), o hospital independente da sua característica de instituição pública, particular ou filantrópica, visa a qualidade no atendimento ao cliente e a ausência de desperdícios ao gerenciar os recursos.

De acordo com ALCALÁ et al. (1982), a qualidade da assistência à saúde mantém relação direta com a quantidade e, principalmente, com a eficácia dos recursos humanos e materiais disponíveis.

CAMPEDELLI et al. (1987) e ROMERO et al. (1994) concordam que existe uma grande dificuldade na mensuração do trabalho da enfermagem que, infelizmente não fornece parâmetros em termos operacionais para cálculos de recursos humanos.

PAIM (1978) critica a tendência que os enfermeiros têm de utilizar a intuição no seu trabalho e a falta de definição de métodos na prática profissional. Apoia o estabelecimento de instrumentos que concretizam e quantificam o trabalho da enfermagem.

Com a finalidade de sanar este problema, autores como ALCALÁ et al. (1982); BACHLE (1985);
SCHULMERICH (1986); CAMPEDELLI et al. (1987); BATTY et al. (1990); KURCGANT (1991); JENNINGS et al. (1989) e ROMERO et al. (1994), elaboraram estudos sobre classificação do grau de dependência de pacientes, estabelecendo critérios de avaliação para o cliente em diferentes categorias, correlacionando as horas de enfermagem trabalhadas.

Os autores, acima citados, estão fundamentados no cuidado progressivo ao paciente que baseia-se na concentração variável de recursos humanos e materiais em torno das necessidades dos clientes, visando uma assistência integral que permite uma adequação entre recursos humanos, materiais e físicos, além de fortalecer o trabalho em equipe.

Os dois métodos mais utilizados para determinar a quantidade e qualidade dos recursos humanos de enfermagem são denominados de tradicional e método progressivo de assistência.

O método tradicional para o cálculo de pessoal descrito por GAIDZINSKI (1991), foi proposto pela Liga Nacional de Educação em Enfermagem dos Estados Unidos, conjuntamente com a Associacion Americana de Hospitales e Associação Americana de Enfermeiras em 1956 e consiste na aplicação da seguinte fórmula:

\section{TFR $=$ HAE x Nº LEITOS x DS + IST JTS}

onde, $\mathbf{T F R}=$ total de funcionários requeridos, $\mathbf{H A E}=$

\footnotetext{
* Professor Auxiliar do Departamento de Enfermagem do Centro de Ciências da Saúde da Universidade Estadual de Londrina e Enfermeira do Hospital Universitário Regional do Norte do Paraná

** Professor Adjunto do Departamento de Enfermagem do Centro de Ciências da Saúde da Universidade Estadual de Londrina e Diretora de Enfermagem do Hospital Universitário Regional do Norte do Paraná
} 
horas da assistência de enfermagem (baseados em parâmetros extraídos da realidade norte americana), $\mathbf{N}^{\mathbf{0}}$ LEITOS = número de leitos, $\mathbf{D S}=$ dias da semana, $\mathbf{J T S}$ = jornada de trabalho semanal e IST = índice de segurança técnico.

Segundo BITTAR (1992) e GAIDZINSKI (1991), este método de planejamento e organização dos serviços hospitalares é baseado na separação dos pacientes por clínica, sexo, grupo etário e, às vezes, de acordo com o nível sócio econômico dos mesmos.

O método de assistência progressiva ao paciente fundamentado no sistema de classificação de pacientes foi introduzido no Brasil por RIBEIRO (1972), e mais recentemente estudos têm sido realizados por ALCALÁ et al. (1982) e ALVES et al. (1988) utilizando a fórmula abaixo para calcular o número de pessoal:

\section{TFR = HAE X $\left(\mathrm{N}^{\circ}\right.$ DE LEITO X \% NA $)+$ IST JTS}

onde, $\mathbf{T F R}=$ total de funcionários requisitados, $\mathbf{H A E}=$ horas da assistência de enfermagem, $\mathbf{N}^{\mathbf{0}}$ DE LEITOS = total de leitos da unidade, JTS = jornada de trabalho semanal, \% $\mathbf{N A}=$ percentual do nivel de atenção referente ao estado ou situação do paciente de acordo com o grau de complexidade das ações desenvolvidas em enfermagem e IST = indice de segurança técnico.

As horas de enfermagem trabalhadas para cada grau de dependência dos pacientes é determinada conforme as várias realidades hospitalares. De acordo com ALCALÁ et al. (1982), a assistência progressiva é preconizada pela Organização Mundial da Saúde, principalmente nas instituições que possuem Centro de Terapia Intensiva, ressaltando que, a classificação de pacientes de acordo com as suas necessidades, já era realizada pelos japoneses há mais de cem anos. $\mathrm{Na}$ Inglaterra, Florence Nightingale quando distribuiu na enfermaria os pacientes do mais ao menos grave, colocando os que exigiam maior atenção mais próximos à enfermeira, praticou o cuidado progressivo ao paciente.

SANTOS (1992) refere que este sistema de classificação oferece a capacidade de prever as exigências ou demanda do cuidado de enfermagem, é considerado clinicamente significativo, objetivo, confiável, fácil de ser atualizado, possibilita identificar as necessidades dos pacientes, além de permitir comparações entre instituições de saúde.

DEIMAN (1994) complementa que o cuidado progressivo ao paciente pode ser aplicado em qualquer instituição de saúde, podendo o enfermeiro adaptá-lo à sua realidade e necessidade da instituição. Sugere que o instrumento de classificação de pacientes deve possuir de três a cinco categorias de forma que o cliente possa ser enquadrado em um destes níveis, que varia desde tratamentos mínimos ou auto-cuidado até terapia intensiva, dependendo da característica da clientela que o hospital possui.

GAIDZINSKI (1991) ressalta que o dimensionamento do pessoal da enfermagem segundo horas trabalhadas, necessita de reconhecimento da situação, caracterização da instituição, da clientela e do serviço de enfermagem, além de sugerir as classificações fundamentadas no cuidado progressivo ao paciente, dividindo-os em seis categorias: cuidados intensivos, intermediários ou semi-intensivo, prolongados, mínimos, ambulatoriais e domiciliares. Cita que outros tipos de denominações também podem ser utilizadas para classificar pacientes como a dependência total, dependência parcial ou incompleta, independência da equipe de enfermagem ou ainda, grau de dependência intensivo, intermediário e auto-cuidado, frisando que o importante é o estabelecimento do perfil do cliente para cada tipo de cuidado ou grau de dependência.

SCHULMERICH (1986) em um departamento de emergência, classificou os pacientes em grupos definidos em grau I, II, III, IV e V, caracterizando as atividades da enfermagem em cuidados diretos e indiretos ao paciente (prontuários, anotações, telefones) e em funções não específicas da enfermagem, ou seja, atribuições que poderiam ser delegadas a outros profissionais. Correlacionou o tempo de permanência do paciente no setor atribuindo grau I àquele doente que permaneceu até 20' após o horário de admissão, grau II até 40', grau III até 60', grau IV até 150 ', grau V até $180^{\prime}$.

ROMERO et al. (1994) baseando-se nas necessidades de mensuração do trabalho da enfermagem criaram em um hospital privado instrumento de classificação do grau de dependência de pacientes para a Unidade de Terapia Intensiva. Utilizaram como parâmetros a alimentação, mobilização, higienização, nível de consciência, eliminações, medicações e procedimentos, verificação de sinais vitais, catéteres, sondas, drenos e procedimentos especiais, atribuindo valores para os itens mencionados, classificando os pacientes em quatro graus de dependência da assistência de enfermagem, semelhante à ALCALÁ et al. (1982); CAMPEDELLI et al. (1987); KURCGANT et al. (1991) e SANTOS (1992).

JENNINGS et al. (1989) confirmaram que esses instrumentos fornecem dados que auxiliam no dimensionamento do quadro de pessoal, monitorizam a produtividade, custos e cobrança do serviço de enfermagem.

BACHLE (1985) elaborou no departamento de urgência, um método de classificação de pacientes, categorizando o atendimento em emergência, urgência e não emergência. 
CAMPEDELLI et al. (1987) fazem uma comparação entre a aplicação dos métodos de classificação do grau de dependência de pacientes sugeridos por ABBOUD (s.d.) e ALCALÁ et al. (1982), onde os índices foram obtidos da realidade de um hospital universitário, levando-se em consideração as horas de enfermagem trabalhadas para realizar o dimensionamento do pessoal da instituição. Concluíram que houve uma grande diferença nos resultados obtidos e que o emprego de um determinado critério pode ser o ponto de partida para efetuar estudos comparativos e para calcular o número do pessoal para o serviço de enfermagem.

SANTOS (1992) realizou um estudo comparativo entre dois métodos de cálculo de pessoal, aplicando a fórmula do método tradicional e a da assistência progressiva de enfermagem a partir de informações estatísticas no atendimento em um hospital universitário, com a finalidade de escolher o método mais adequado. Concluiu que o método de assistência progressiva é mais viável, desde que seja efetuado um estudo em termos de ocupação de leitos, horas de enfermagem por tipo de cuidado e desempenho do pessoal.

Baseados nos conceitos acima levantados, em 1995, em um hospital universitário público do Norte do Paraná foi elaborado e implantado pelos autores da pesquisa, um impresso que classificou os pacientes em quatro graus de dependência da enfermagem. Após um ano de uso do instrumento, verificou-se que em algumas unidades havia variação na observação dos enfermeiros nos diferentes turnos, o que motivou a elaboração desta pesquisa.

Portanto, o objetivo deste trabalho é validar o instrumento de classificação da dependência dos pacientes em relação a assistência de enfermagem em um hospital escola público.

\section{METODOLOGIA}

O estudo foi realizado no HOSPITAL UNIVERSITARIO REGIONAL DO NORTE DO PARANÁ (HURNP), situado na cidade de Londrina/Pr, após ter sido submetido a avaliação e aprovação pela comissão de ética e obtido o consentimento de participação na pesquisa pelos membros envolvidos. $\mathrm{O}$ hospital mantém convênio com o Sistema Único de Saúde e dispõe de 294 leitos de internação, distribuídos entre as unidades médico-cirúrgicas feminina e masculina, pronto socorro, centro de terapia intensiva, moléstias infecto-contagiosas, tisiologia, maternidade, berçário, pediatria, UTI neonatal, UTI pediátrica, entre outras.

Foi utilizado como campo de pesquisa as unidades de internação médico-cirúrgicas feminina e masculina com capacidade de 43 e 73 leitos respectivamente, unidade pediátrica com 30 leitos e centro de terapia intensiva que comporta 17 pacientes.

Participaram do trabalho os enfermeiros que atuam nestas unidades no período matutino, vespertino e noturno A e B, totalizando 50 profissionais.

O instrumento (Anexo 1), após sua elaboração, foi aplicado no período de um ano, quando observou-se através da análise dos resultados que havia discordância nos dados obtidos, por falta de padronização nos critérios de preenchimento do impresso. Para identificar as causas das variações, os enfermeiros foram entrevistados informalmente pelo autor da pesquisa. Alegaram que a nomenclatura utilizada propiciava duas interpretações, ocasionando classificações em graus diferentes quando o instrumento era aplicado por mais de um enfermeiro. Nesta entrevista os profissionais solicitaram reformulações no instrumento e que fosse realizado um treinamento antes que o novo modelo fosse aplicado.

A solicitação foi aceita e o segundo instrumento utilizado é composto por quatro graus de classificação do paciente em relação à dependência de enfermagem, conforme Anexo 2.

No treinamento realizado para os enfermeiros, foi discutido e analisado o novo instrumento, comparando-o com o primeiro, esclarecendo as dúvidas e definindo os critérios que seriam adotados para classificar os pacientes nos quatro graus de dependência da enfermagem.

Dentre os critérios, foi determinado que para um paciente se enquadrar em qualquer grau de dependência, ou seja, grau I, II, III e IV, ele dependeria dos serviços da enfermagem em pelo menos três itens. Foi consenso entre o grupo, que os pacientes em pré-operatório seriam classificados como grau de dependência II e que em pósoperatório imediato seriam classificados como grau de dependência III, passando para grau II no $1^{\circ}$ pósoperatório, desde que não tivessem sido submetidos a uma cirurgia complexa. Definiu-se que terapia endovenosa intensiva é quando o paciente recebe mais de três tipos de administração endovenosa contínua com ou sem bombas de infusão.

Ficou determinado que cirurgias complexas são aquelas de grande porte, que requerem preparo de cólon no pré-operatório ou aquelas onde são realizados os transplantes.

Definiu-se que de acordo com as características dos pacientes internados na unidade de pediatria, acompanhados ou não pelos seus pais, todos seriam classificados nos graus III e IV.

O instrumento foi aplicado pelos enfermeiros dos quatro períodos nas unidades mencionadas anteriormente, durante o período de um mês, como parte prática do 
treinamento. Após esta etapa, 7 enfermeiros com experiência profissional acima de dois anos e que participaram do treinamento proposto, foram solicitados para validar o instrumento.

Neste processo de validação, os enfermeiros classificaram os pacientes simultaneamente, ou seja, se reuniram nas unidades em horário e local determinado e, seqüencialmente, mas individualmente, um seguido do outro, passaram visita leito por leito realizando o exame físico e avaliando as condições gerais do paciente para classificá-lo de acordo com os graus de dependência, iniciando-se o processo pelo enfermeiro controle, que é o autor desta pesquisa. Durante esta etapa os enfermeiros não trocaram informações entre si.

A aplicação do instrumento foi estipulada para o período matutino devido ser este o horário que o enfermeiro revisa os planos de assistência de enfermagem, fazendo o exame físico e preenchendo o boletim de informação aos familiares dos pacientes.

Os dados estatísticos foram calculados através do percentual de concordância global e por grau de dependência. Foi também aplicado o coeficiente Kappa, baseando-se em KRAMER \& FEINSTEIN (1981), somente nas análises dos resultados obtidos nas unidade de internação médico cirúrgica, ala feminina e masculina, por possuírem muitos leitos e por internarem pacientes de várias especialidades médicas, o que possibilita a variação na interpretação dos graus de dependência pelos enfermeiros.

Baseando-se nestes autores, considerou-se como grau de concordância regular os resultados obtidos abaixo de $60 \%$, moderada entre 60 e $74 \%$ de concordância com o enfermeiro controle, 75 a $84 \%$ foi considerado uma concordância forte e resultados entre 85 e $100 \%$ foi considerado um grau de concordância quase perfeita.

\section{RESULTADOS E DISCUSSÕES}

Foram classificados 97 pacientes da unidade de internação médico-cirúrgica incluindo ala feminina e masculina, 30 pacientes da pediatria e 17 pacientes do centro de terapia intensiva, totalizando 144 doentes.

O número de pacientes analisados e a técnica de validação do instrumento permitiram ao autor participar ativamente do processo, comparando a sua classificação com a dos enfermeiros que atuavam nas unidades onde $o$ estudo foi realizado.

DEIMAN (1994) reforça que o instrumento empregado para classificar e categorizar os pacientes deve ser testado para detectar sua eficácia e validez com a finalidade de assegurar a sua exatidão. A confiabilidade do instrumento variará de acordo com a uniformidade do grau de dependência na classificação do paciente realizada por dois ou mais enfermeiros simultaneamente, ou seja, quanto maior o número de enfermeiros chegar ao mesmo resultado, melhor será o instrumento. Sugere que para o instrumento ser considerado confiável deve haver $80 \%$ de acordo entre os classificadores.

O mesmo autor descreve que para fazer o cálculo do número pessoal de enfermagem em um hospital, podese utilizar o sistema de classificação de pacientes, ressaltando que classificar doentes, significa dividi-los em grupos conforme as necessidades que requerem da enfermagem.

Esta classificação dos pacientes auxilia no desenvolvimento do processo de enfermagem esquematizado por ser ordenador e direcionador do trabalho da enfermagem, permitindo uma avaliação dos procedimentos que estão sendo realizados por estes profissionais.

Atualmente a enfermagem tem sentido necessidades, cada vez maiores, de utilizar o método científico como estrutura para a organização da assistência de enfermagem, fazendo com que os profissionais desenvolvam métodos práticos, eficientes e rápidos de obterem resultados, relacionados à melhoria da assistência e ao dimensionamento de pessoal.

Foi com esta finalidade que o primeiro instrumento foi criado e implantado nesta instituição, porém houve necessidade de reformulações para tornálo um instrumento facilitador e mensurador do trabalho da enfermagem.

BATTY et al. (1990), em estudo semelhante de classificação de pacientes por um período de quatro semanas, também encontraram problemas com o método, pois os resultados não corresponderam às expectativas, sendo necessário a reformulação do instrumento e validação do mesmo.

Os mesmos autores descrevem que o processo de validação do instrumento Rush-Medicus que funciona como sistema de classificação de pacientes, é extenso e determinado pela observação dos enfermeiros em atividade, sendo que enfermeiros especialistas deram assistência de consultoria e orientação no processo.

Para o desenvolvimento desta pesquisa foi necessária a assessoria de um estatístico para analisar e possibilitar ou não a validação do instrumento classificatório da dependência do paciente em relação ao serviço de enfermagem.

Analisando os dados obtidos verificou-se que, embora os enfermeiros das unidades de internação médico-cirúrgicas tivessem sido submetidos a um treinamento onde foram estabelecidos critérios para classificar os pacientes, ainda existiu variação nos resultados estimados conforme apresentado na Figura 1. 


\begin{tabular}{|l|c|c|c|c|c|c|c|}
\hline & ENF 1 & ENF 2 & ENF 3 & ENF 4 & ENF 5 & ENF 6 & ENF 7 \\
\hline Grau I & $45 \%$ & $73 \%$ & $64 \%$ & $100 \%$ & $100 \%$ & $100 \%$ & $100 \%$ \\
\hline Grau II & $86 \%$ & $100 \%$ & $95 \%$ & $81 \%$ & $70 \%$ & $89 \%$ & $97 \%$ \\
\hline Grau III & $75 \%$ & $100 \%$ & $75 \%$ & $76 \%$ & $81 \%$ & $90 \%$ & $67 \%$ \\
\hline Grau IV & $100 \%$ & $100 \%$ & $100 \%$ & $100 \%$ & $100 \%$ & $100 \%$ & $100 \%$ \\
\hline Global & $\mathbf{7 5} \%$ & $\mathbf{9 3} \%$ & $\mathbf{8 5} \%$ & $\mathbf{7 5} \%$ & $\mathbf{7 5} \%$ & $\mathbf{8 5} \%$ & $\mathbf{8 5} \%$ \\
\hline
\end{tabular}

FIGURA 1 - Comparação do percentual de concordância global e por grau de dependência entre o enfermeiro controle e os enfermeiros da unidade de internação médico-cirúrgica

Observou-se que quatro enfermeiros concordaram em $100 \%$ com o enfermeiro controle, em classificar os pacientes como grau I. O enfermeiro 2 concordou em $73 \%$, atingindo um nível de concordância moderada. E em concordância regular e moderada os enfermeiros 1 e 3 apresentaram índices de $45 \%$ e $64 \%$, respectivamente.

O grau II obteve uma concordância variável entre $70 \%$ e $100 \%$, ficando 5 enfermeiros na faixa dos $86 \%$ e $100 \%$, o que demonstra um nível de concordância quase perfeito.

No grau de dependência III somente o enfermeiro 7 obteve um percentual inferior a $70 \%$ e os demais enfermeiros ficaram entre 75 e $100 \%$, o que também revela um grau de concordância forte com o enfermeiro controle na classificação dos pacientes.

Observou-se que 7 enfermeiros atingiram 100\% de concordância com o enfermeiro controle no grau IV, o que evidencia um bom discernimento dos mesmos em relação a este grau.

Verificou-se, também, que o instrumento não deixa dúvidas em relação a classificação dos pacientes graves ou totalmente dependentes da enfermagem, pois os itens estão bem definidos, da mesma forma como as características do paciente.

O grau de concordância global, ou seja, levando em consideração todos os graus de dependência ficou em $75 \%$ para o enfermeiro 1 e 5 , em $78 \%$ para o enfermeiro 4, 85\% para os enfermeiros 3, 6 e 7, o enfermeiro 2 atingiu 93\%. Observou-se que todos enfermeiros tiveram um percentual de concordância superior à $75 \%$, o que demonstra uma forte correlação nos dados obtidos.

Esta variação pode ter ocorrido pelo fato destas unidades comportarem muitos leitos e internarem pacientes de várias especialidades médicas.

Segundo SILVA \& ARAÚJO (1994), “a maneira da enfermeira encarar o mundo influencia a maneira de olhar o paciente, ou seja, o que embasa a compreensão de um paciente e o que fazer com relação a seu estado de saúde, depende da bagagem teórica que a profissional possui". Ressaltam que uma das dificuldades encontradas, tanto pelos acadêmicos como pelos profissionais da enfermagem, tem sido a realização de um levantamento ou coleta de dados consistente que permita um conhecimento adequado do indivíduo.

Verificou-se que, embora o instrumento tenha sido implantado após um treinamento prévio e com ampla discussão dos critérios de classificação, ainda houve algumas divergências na categorização dos graus de dependência, demonstrando que o processo de classificação dos pacientes também depende da experiência profissional e análise pessoal do enfermeiro.

SILVA \& ARAÚJO (1994) ressaltam que a enfermeira e o paciente possuem características pessoais que interferem na percepção das informações, hábitos e limitações vindas de preconceitos e estereótipos. Lembra que as diferenças individuais de percepção podem ser entendidas em termos do campo psicológico de cada um e em termos das necessidades e metas de cada pessoa.

CUNHA et al. (1994) descrevem que a ação do enfermeiro é prejudicada pela falta de propedêutica das ciências básicas que fundamentam a área da saúde, tornando a própria ação e a tomada de decisão dos enfermeiros prejudicada. A deficiência de conhecimento a nível das ciências básicas propicia numa avaliação incompleta dos problemas de enfermagem apresentados pelos pacientes resultando num número de ações insuficientes para o atendimento. Acrescentam que outro fator a ser considerado é a falta de segurança do enfermeiro em relação à sua própria atuação tanto no ato de prescrever como no seu conteúdo, gerando dúvidas.

DEIMAN (1994) reforça que a maneira mais eficaz para se conseguir uma boa homogeneidade na interpretação e aplicação do instrumento entre os enfermeiros classificadores, é mediante um programa de treinamento em serviço para todos os participantes. Outro parâmetro é observar se a categoria que o paciente está sendo enquadrado corresponde às horas de assistência que ele está exigindo da enfermagem. 
A Figura 2 apresenta o nível de concordância global na classificação dos pacientes feita pelos enfermeiros em relação ao enfermeiro controle.

\begin{tabular}{|c|c|c|c|c|}
\hline ENFERMEIROS & REGULAR & MODERADA & FOR TE & $\begin{array}{c}\text { QUASE } \\
\text { PERFEITA }\end{array}$ \\
\hline$E_{\text {if } I} I$ & & & $\mathrm{x}$ & \\
\hline$E$ If 2 & & & & $\mathrm{x}$ \\
\hline$E_{n f} 3$ & & & & $\mathrm{x}$ \\
\hline$E v_{f} 4$ & & & $\mathrm{x}$ & \\
\hline$E x f^{2}$ & & & $\mathrm{x}$ & \\
\hline$E x f 6$ & & & & $\mathrm{x}$ \\
\hline$E_{n f} f$ & & & & $\mathrm{x}$ \\
\hline
\end{tabular}

FIGURA 2 - Resultados de concordância global encontrado nos enfermeiros da unidade médicocirúrgica comparando com o enfermeiro controle para classificação de pacientes

Verificou-se no Centro de Terapia Intensiva que os enfermeiros, incluindo o enfermeiro controle, classificaram todos os pacientes no grau IV, obtendo um resultado de $100 \%$ de concordância. Isto reforça a opinião que para os pacientes graves não há necessidade de aplicação do instrumento.

Considerando que este hospital é regional e a ocupação dos leitos do Centro de Terapia Intensiva é de $95 \%$, observou-se que quando o paciente apresenta melhora, recebe alta do setor, motivo pelo qual dificilmente encontram-se doentes classificados em outros graus.

No resultado obtido na unidade pediátrica, observou-se $100 \%$ de concordância dos enfermeiros em classificar as crianças internadas como grau III e IV.

$\mathrm{Na}$ unidade pediátrica, durante o processo de avaliação do instrumento, ocorreu uma longa discussão entre os enfermeiros sobre a classificação dos graus de dependência das crianças. Foi questionado se o fato da criança estar com acompanhante ocasionaria alteração na atuação da enfermagem, chegando-se ao consenso de que o acompanhante ameniza a internação da mesma, visto que esta se encontra em ambiente desconhecido com pessoas diferentes do seu cotidiano, muitas vezes irritada e chorosa e sujeita a procedimentos invasivos, desde que o acompanhante esteja orientado e envolvido o suficiente no tratamento da criança, necessitando para isso de orientação e auxílio constante da equipe de enfermagem.

Outra observação dos enfermeiros desta unidade é que dependendo da patologia e sintomatologia, existe uma descompensação rápida no estado geral, necessitando de observações freqüentes da enfermagem para detectá-las precocemente evitando a evolução da doença.

OKAYAMA et al. (1996) afirmaram que para prestar assistência de enfermagem à criança é preciso geralmente de duas pessoas, a mãe e um profissional da enfermagem ou de dois profissionais, porque há necessidade de distrair a criança durante os procedimentos para que não haja intercorrências ou alterações nos resultados esperados. Deixar a criança manusear os objetos reais que farão parte da sua assistência como estetoscópio, esfigmomanômetros entre outros, faz com que ela se familiarize com o tratamento; no entanto, para isto é necessário que haja disponibilidade de tempo e habilidade do pessoal de enfermagem, proporcionando distração e brincadeiras, envolvendo a criança para a realização de um procedimento com sucesso.

Embora no centro de terapia intensiva e unidade pediátrica todos os pacientes tenham sido classificados no grau IV de dependência da enfermagem, os motivos que levaram a este resultado são diferentes conforme descrito anteriormente. Ressalta-se que há uma diferença nas horas de enfermagem trabalhadas com adultos e crianças, sendo necessário elaborar um instrumento específico para classificar o grau de dependência da assistência de enfermagem dos pacientes pediátricos.

ALCALÁ et al. (1982) distribuíram as horas de enfermagem trabalhadas para pacientes adultos que necessitam de cuidados mínimos em 2,5 horas e crianças em 4,5 horas, para cuidados intermediários atribui 4,5 horas para adulto e 5,5 horas para crianças, cuidados semiintensivos 10 horas para adultos e também para crianças, e cuidados intensivos 18 horas tanto para adultos como para crianças.

RAMOS et al. (1994) realizaram estudos comparativos de tempo versus movimento do trabalhador, com a finalidade de obter o tempo gasto de enfermagem com cada paciente. Observaram que na prática o tempo gasto para a realização de um determinado procedimento oscila de acordo com influências intrínsecas (idade, complexidade e colaboração do paciente) e extrínsecas (material disponível, destreza do funcionário) ao paciente.
$\mathrm{O}$
CONSELHO
FEDERAL
$\mathrm{DE}$

ENFERMAGEM (1996) através da Resolução 189 atribuiu 3,0 horas de enfermagem trabalhadas para o paciente que necessita de assistência mínima ou autocuidado, 4,9 horas para assistência intermediária, 8,5 horas para assistência semi-intensiva, 15,4 horas para assistência intensiva, considerando-se as horas de enfermagem por leito nas 24 horas.

Observou-se neste estudo que, embora tenha ocorrido pequena variação nos resultados esperados, o grau de concordância global forte e quase perfeito predominaram, o que permite a utilização do instrumento 
para a caracterização da clientela assistida e também ser utilizado para calcular o número de pessoal de enfermagem.

\section{CONCLUSÕES}

- O instrumento de classificação dos pacientes segundo o grau de dependência da enfermagem, após reformulação, foi validado e mostrou-se compatível com a realidade do hospital, categorizando os pacientes internados de acordo com o grau de dependência.

- A validação do instrumento proporcionou aos enfermeiros uma análise profunda no processo de observação das necessidades dos pacientes, tornandose uma ferramenta de trabalho eficiente na prática diária.

- O novo instrumento de classificação dos pacientes é de fácil e prática aplicabilidade permitindo homogeneização do entendimento pelos enfermeiros dos diferentes graus de dependência do paciente em relação à assistência de enfermagem.

- Para classificar pacientes pediátricos recomenda-se a elaboração de um instrumento específico.

- Nas unidades de Terapia Intensiva dispensa-se a aplicação do instrumento pelo fato de todos pacientes serem classificados em grau IV.

\section{VALIDATION OF AN INSTRUMENT THAT CLASSIFIES PATIENTS IN FOUR LEVELS OF DEPENDENCE TO NURSING CARE}

This study presents the process of validation of a tool that classifies patients in four levels of dependence to the nursing care delivered at a University Hospital. The research gave to participants an opportunity to analyse patients' needs, showing the instrument as an efficient tool for nursing practice.

\section{ELABORACIÓN DE UN INSTRUMENTO QUE CLASIFICA A LOS PACIENTES EN CUATRO GRADOS DE DEPENDENCIA DE CUIDADOS REALIZADOS POR ENFERMEROS}

Esta investigación presenta el proceso de elaboración de un instrumento que clasifica a los pacientes en cuatro grados de dependencia de cuidados realizados por enfermeros en un hospital universitario público. El estudio ha proporcionado a los enfermeros participantes, la oportunidad de analizar las necesidades de los pacientes, constituyéndose el instrumento en una herramienta de trabajo importante en la práctica de la enfermería.

TERMINOS CLAVES: cuidados de enfermería, paciente, cuestionario

\section{Anexo 1}

\begin{tabular}{|c|c|}
\hline $\begin{array}{c}\text { GRAU DE } \\
\text { DEPENDENCLA }\end{array}$ & CARACTERISTICAS DO PACIENTE \\
\hline I & $\begin{array}{l}\text { - Sem desvios de comportamento. } \\
\text { - Deambula, toma banho e alimenta-se } \\
\text { sozinho. } \\
\text { - Tratamentos mínimos. } \\
\text { - Controle de sinais vitais e eliminações três } \\
\text { vezes ao dia. } \\
\text { - Orientação e supervisão do auto-cuidado. }\end{array}$ \\
\hline
\end{tabular}

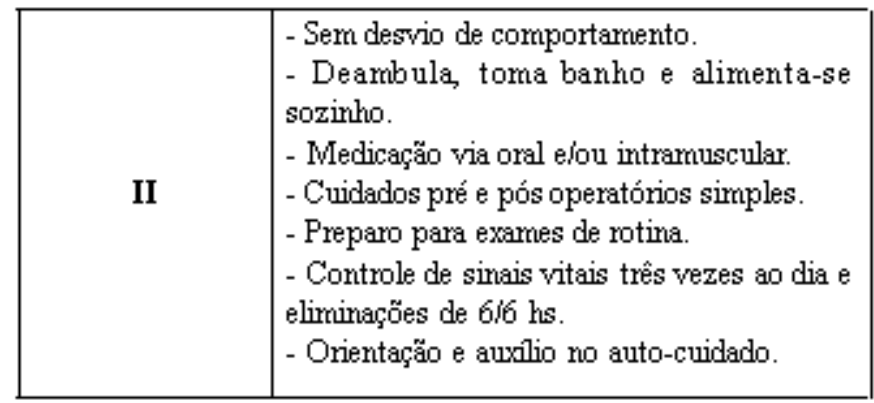

\begin{tabular}{|c|c|}
\hline $\begin{array}{c}\text { GRAU DE } \\
\text { DEPENDENCLA }\end{array}$ & CARACTERISTICAS DO PACIENTE \\
\hline III & $\begin{array}{l}\text { - Com ou sem desvios de comportamento. } \\
\text { - Necessita de auxilio na deambulação, no } \\
\text { banho e na alimentação. } \\
\text { - Medicação intramuscular, subcutânea elou } \\
\text { endove nosa. } \\
\text { - Cuidados pré pós operatónios complexos. } \\
\text { - Controle de sinais vitais e eliminaçóes de } \\
4 / 4 \text { hs. } \\
\text { - Observações frequientes das condições } \\
\text { gerais do paciente. }\end{array}$ \\
\hline IV & $\begin{array}{l}\text { - Inconsciente ou com desvios no padrão de } \\
\text { comportamento. } \\
\text { - Acamado continuamente, higiene no leito. } \\
\text { - Alimentação por sondas ou nutrição } \\
\text { parenteral total. } \\
\text { - Terapia endovenosa intensiva. } \\
\text { - Controle de sinais vitais de } 2 / 2 \mathrm{hs.} \\
\text { - Requer medidas complexas para } \\
\text { manuterção da vida. } \\
\text { - Total dependência da enfermagem (incluir } \\
\text { crianças). }\end{array}$ \\
\hline
\end{tabular}


Anexo 2

CLASSIFICAÇÃO DO GRAU DE DEPENDÊNCLA DO PACIENTE EM RELAÇÃO AO ATENDIMENTO DE ENFERMAGEM

\begin{tabular}{|c|c|}
\hline GRAU DE DEPENDÊNCIA & CARACTERÍSTICAS DO PACIENTE \\
\hline I & $\begin{array}{l}\text { - Deambula sem auxilio. } \\
\text { - Toma barho e alimenta-se sozinho. } \\
\text { - Sem desvios de comportamento. } \\
\text { - Orientação e supervisão do auto-cuidado. }\end{array}$ \\
\hline
\end{tabular}

\begin{tabular}{|l|l|}
\hline & - Necessita ou não de auxilio na deambulação, no barho e na alimentação. \\
& - Quantidade moderada de medicação, terapia endovenosa de rotina. \\
& - Cuidados pré e pós opertóno de rotina. \\
& - Sern desvio de comportamento. \\
& - Orientação e auxilio no auto-cuidado. \\
\hline
\end{tabular}

\begin{tabular}{|l|l|}
\hline & - Necessita de auxilio na deambulação, no barho e na alimentação. \\
& - Grande quantidade de medicação endovenosa. \\
& - Cuidados pré e pós operatónos complexos. \\
& - Com ou sem desvios de comportamento. \\
& - Observações freqüentes das condições gerais do paciente. \\
\hline
\end{tabular}

\begin{tabular}{|l|l|}
\hline & - Acamado continuamente. \\
& - Higiene no leitolalimentação por tubos ou nutrição parenteral total. \\
& - Requer medidas complexas para manutenção da vida. \\
& - Terapia endovenosa intersiva. \\
& - Complicação pós-operatónas. \\
& - Inconsciente ou com desvios no padrão de comportamento. \\
\hline
\end{tabular}

\section{REFERÊNCIAS BIBLIOGRÁFICAS}

\section{ABBOUD, M. Dotação de pessoal na organização} hospitalar. São Paulo: Escola de Administração de Empresas de São Paulo da FGV/HC/FMUSP, s.d. 9p. (apostila)

02. ALCALÁ, M.U. et al. Cálculo de pessoal: estudo preliminar para estabelecimento de quadro de pessoal de enfermagem na superintendência médico Hospitalar de Urgência. São Paulo: Prefeitura Municipal de São Paulo, 1982. p.1-46.

03. ALVES, S.M. et al. Enfermagem: contribuição para um cálculo de recursos humanos na área. Rio de Janeiro: Coordenadoria de Comunicação Social do INAMPS, 1988.

04. BACHLE, M. An effective method to determine nursing workload. Health Care, v. 27, n. 6, p. 21-25, Sept. 1985.

05. BATTY, K. et al. Patient classification: a visible difference. Nurs. Mangement, v. 21, n. 9, p. 7175, Sept. 1990.

06. BITTAR, O.J.N. Dimensionamento de pessoal na área hospitalar. Previdência em dados, Rio de Janeiro, v. 7, n. 1, p. 10-18, jan-mar. 1992.

07. CAMPEDELLI, M.C. et al. Cálculo de pessoal em enfermagem: competência da enfermeira. Rev. Esc. Enfermagem USP, São Paulo, v.1, n.21, p.313, abr. 1987.
08. CONSELHO FEDERAL DE ENFERMAGEM. Documentos básicos, Brasília, 1996. p. 374-384.

09. CUNHA, I.C.K.O. et al. Sistema de apoio à decisão em enfermagem - pré avaliação. In: ENFTEC, 4, São Paulo, 1994. Anais. São Paulo, 1994. p. 156160.

10. DEIMAN, P. Dotacion de personal para los servicios de enfermeria en hospitales de distrito para la atencion primaria de salud. Washington: OMS, 1994. p. 34-48. (Serie PALTEX para ejecutores de Programas de Salud, 32).

11. GAIDZINSKI, R.R. Dimensionamento de pessoal de enfermagem. In: KURCGANT, P. et al. Administração em enfermagem. São Paulo: EPU, 1991. Cap.7, p. 91-96.

12. JENNINGS, M.B. et al. Selecting, implementing and evolution patient classification systems: a mensure of productivity. Nurs. Admin. Q., v. 14, n. 1. p. 24-35, 1989.

13. KRAMER, M.S.; FEISTEN, A.R. Clinical biostatistics LIV. The biostatistics of concordance. Clin.Pharmacol. Ther., v. 1, n. 29, p. 111-122, 1981.

14. KURCGANT, P. et al. Administração em Enfermagem. São Paulo: EPU, 1991. 
15. OKAYAMA, M.S. et al. Comparação entre os resultados obtidos no tempo despendido na prestação de cuidados à criança e ao adulto na unidade de pediatria do Hospital Sírio Libanês. In: ENFTEC, 5, São Paulo,1996. Anais. São Paulo.1996. p. 482-485.

16. PAIM, L. Problemas, prescrições e planos. Cad. Científico ABEN, n. 1, p. 70-73, 1978.

17. RAMOS, M.L.T. et al. Sistema de classificação de pacientes por grau de dependência do cuidado de enfermagem: ENFSCORE. In : ENFTEC, 4, São Paulo, 1994. Anais. São Paulo, 1994. p. 128133.

18. RIBEIRO, C.M. Sistema de classificação de pacientes como subsídio para provimento de pessoal de enfermagem. São Paulo, 1972. Tese (Doutorado) - Escola de Enfermagem, Universidade de São Paulo.
19. ROMERO, E. et al. Proposta de um instrumento para classificação dos cuidados de enfermagem quanto ao grau de dependência. In: ENFTEC, 4, São Paulo, 1994. Anais. São Paulo, 1994. p. 482-485.

20. SANTOS, S.R. Cálculo de pessoal em enfermagem: estudo de dois métodos. Rev. Esc. Enfermagem USP, v. 28, n. 2, p. 137-154, ago. 1992.

21. SILVA, M.J.P.; ARAÚJO T.L. de. Repensando a entrevista de coleta de dados. In: ENFTEC, 4, São Paulo, 1994. Anais. São Paulo, 1994. p. 541546.

22. SCHULMERICH, S.C. Coverting patient classification data into staffing requirements for the emergency departament. J. Emergency Nurs., v. 12, n. 5, p. 286-290, Sept/Oct. 1986. 